\title{
Detection of Abnormal Movements of a Crowd in a Video Scene
}

\author{
G. Mariem, E. Ridha, and Z. Mourad
}

\begin{abstract}
There are many applications for the detection of anomalies; in this paper we propose a new method for the detection of abnormalities in crowded scenes. In our method, we present a hand technique for temporal tracking of different people during movement in a video sequence, using the technique of Gaussian mixture model (GMM). This method is based on the blob detector analyzing foreground functioning of cells based on which is created a statistical modelling of the individual item. The Gaussian mixture model (GMM) is used as a position vector to extract different motion characteristics. In addition to detecting the abnormal behavior of the crowd we propose a new simple method. We use the differential method of Lucas and Kanade to estimate abnormal events observed in a surveillance video. It presents an algorithm to accelerate the process of abnormal motion detection based on a local adjustment of the velocity field by calculating the light intensity between two images to detect the abnormal movement.
\end{abstract}

Index Terms-Anomaly detection, crowd analysis, Gaussian mixture model, pyramids of Lucas and Kanade, video surveillance.

\section{INTRODUCTION}

Surveillance cameras invaded all public areas. These cameras require a continuous human presence for monitoring the captured scenes and intervene when necessary or to alert officials in case of problems. Thanks to the large number of cameras, automated monitoring and intervention scenes has become paramount. For this, it was thought to provide a tool which will accelerate the detection of abnormal movements in video scenes. But there are many applications of surveillance systems that can generally prevent crime and to make social control such as crowd movement and monitoring of traffic flows and more companies use monitoring systems since they include automatic video processing. To detect abnormal events many innovations in video surveillance has been made in recent years in areas such as the temporal object tracking [1], detection of persons [2], traffic monitoring [3] and detection of a host [4]. There are several methods to detect moving objects in video surveillance.

Several works have been proposed for abnormal events divided into two: spatio-temporal methods and statistical methods. As a model for the monitoring and detection of

Manuscript received December 10, 2014; revised June 16, 2015. This work was supported by Research Groups on Intelligent Machines (REGIM), University of Sfax National Engineering School of Sfax (ENIS).

G. Mariem is with Higher Institute of Computer Science and Multimedia of Gabes (ISIMG), Gabes, Tunisia (e-mail: mariem21 gnouma@ gmail.com).

E. Ridha and Z. Mourad are with Research Groups on Intelligent Machines (REGIM), University of Sfax National Engineering School of Sfax (ENIS), Tunisia (e-mail: ridha_ejbali@ieee.org, mourad.zaied@ieee.org). anomalous events based on spatio-temporal methods can find the histogram of optical flow (HOF), the spatio-temporal gradient [5], the force social model [6], The chaotic invariant [7] and th mixtures of dynamic textures [8].

Four statistical methods we can found: Mixture Principal Component Analysis (MPPCA) [9], the use of Hidden Markov Model (HMM) for the detection of abnormal events [10], and the domain Markov model (MRF) to detect activity. Anomalous events can be divided into two events in the crowd scenes crowded and those in the deserted scenes. We are interested in this paper to present the events in a big crowd. In general, for crowded scenes, many objects that occur simultaneously where we had difficult to segment and detect the different events that happen, which is why most methods are based on the typical abnormal. For example, for detecting certain types of rare events, Adam et al. [11] provides histograms based on the probability of measuring the optical flow algorithm. Also Mehran and the authors [7] present a new method to locate and detect abnormal behavior in several videos of the crowd on the model of social work. Another work proposed by Irani and Boiman [12] on the detection of abnormalities that include various analyzes of the crowd involved in the search for methods of computer vision.

In this article, we will propose two methods one for temporal tracking movements of individuals in a crowd and the other for the detection of abnormal movements. We work on three stages that require prior background segmentation, no prior learning of normal movement, with recognition of abnormal movement and tracking of moving object. We analyze the trajectory for the individual detection method of improving the Gaussian mixture model (GMM) and the method of Lucas Kanade pyramid for anomaly detection.

\section{Detection of AnOMAly IN CROWd Scene}

We begin by providing a method for the detection and monitoring of temporal crowd then propose abnormality detector.

\section{A. Crowd Detection}

Despite the existence of multiple and different approaches to the problem of tracking in crowds, their applications remain a challenge. Indeed, in scenes that problem appears more crowded than in uncluttered scenes, for example, the small size of the target object or occlusions with other objects are factors that make it very difficult to detect target objects, and this justifies why the monitoring and detection in congested places fail.

To solve this problem, several solutions have been proposed as in [6] in which the authors have replaced those 
with particles tracking. Other authors have proposed algorithms that eliminate the foreground and see it as a region of interest [13].

Our proposal for the detection and monitoring of the crowd temporal approach is presented. First, various characteristics have to retrieve the contents of each lead frame running. Subsequently, we use the algorithm for Gaussian Mixture (GMM for each object detected. In the rest of section we will describe each of the system.

\section{B. Overview of Our Method}

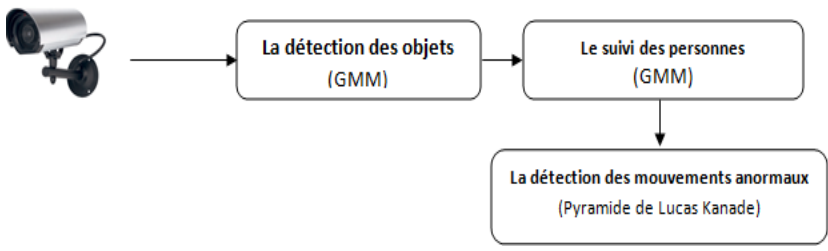

Fig. 1. Proposed approach for the detection of abnormal.

Unlike the approaches mentioned above (see Fig. 1) for the detection of abnormal movement in a crowd, we will detect abnormal behavior in a broad sense and not accurate eg unexpected movement of people. We will aim to develop techniques that are generally applicable to various surveillance videos.

Due to the sudden changes in scenes such as lighting or sudden climate change and darkness, motion detection is a difficult problem to treat, that is why we propose a solution based on a Gaussian mixture (GMM) applied to each image location, to model the local distribution of the intensities image. To detect various objects in cluttered scenes. Detecting persons in these scenes is based on the detection of each individual's movement, then adapting our method to the detection and monitoring of the time people in the scene beginning with the segmentation of the objects from the background (GMM) then switching to the use of morphological opening for removing noise and extracting the boxes containing the connected components for each moving element by using the object vision.BlobAnalysis.

The background subtraction is frequently used to detect moving regions by subtracting the original image I pixel by pixel of an image reference background technique. After creating the foreground, a morphological filter is applied to reduce the effect of noise. The main subtraction of the background goal is to identify the objects and the development of a robust background model to environmental changes.

To model each pixel background before we use a mixture of three Gaussian as present in [14], in this context the three Gaussian fit in the shade, people, and street. The model is initialized using the EM algorithm. Thereafter each Gaussian follows one brand: the component that has the largest variance is classified as individuals and the rest are classified as street.

After detecting the background proceeds to step detecting foreground in this step, we identify the pixels of the image I by comparing each pixel $\{X 1 \ldots X t\}$ with each Gaussian. A Gaussian mixture model can be formulated as follows: Each pixel is characterized by its intensity in the RGB color space. Then the probability is calculated from the observed value of each pixel:

$$
P\left(X_{t}\right)=\sum_{i=1}^{k} \omega_{i, t} \eta\left(X_{t} ; \mu i, t, \sum_{i, t}\right.
$$

where

$$
\sum_{i=1}^{k} \omega_{i, t}=1
$$

The mean of the Gaussian mixture is:

$$
\mu_{t}=\sum_{i=1}^{k} \omega_{i, t} \mu_{i, t}
$$

With

$K$ : the number of distributions

$\omega_{i, t}:$ the weight of the $i$ th Gaussian

T: the time

$\sum_{i, t}:$ the standard deviation

Then, we find the bounding boxes for each connected component corresponding to a movement of persons with the object vision.BlobAnalysis. The blob analysis is the identification and study of the regions of connected pixels in an image.

To construct the limits of the rectangle following people during movement we must find the position of each individual during movement by using the result of the bounding box (BBOX) object tracking.

The position is defined as [ $x y$ width height]

$x, y$ : the upper left point of the rectangle

$x$ : minimum of $x$

$y$ : maximum of $y$

Width: $[x, y+H ; X 2+L, y+H]$

Height: $[x, Y 2 ; x, y+H]$

$H$ : height of rectangle

$L$ : width of rectangle

$X 2$ : maximum of $x$

$Y 2$ : minimum of $y$

\section{Detection of Abnormal Events}

In this article, we improvers a method to detect abnormal movements of the crowd by using the differential method of Lucas and Kanade (see Fig. 2).

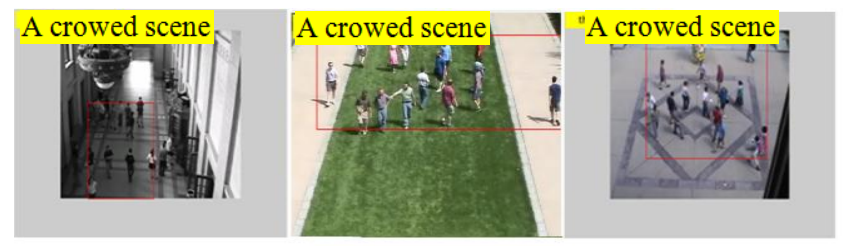

Fig. 2. Results of crowd detection.

This method calculates the minimum of the quadratic matching function (SSD), this function allows the estimation of the parameters of a transformation affecting all parts of the image. The movement calculation is to associate each pixel $(x, y, t)$ a vector $\left(v_{x}^{t}, v_{y}^{t}\right)$ representing the projection onto the plane of the velocity vector $\left(v_{x}{ }^{t}, v_{y}{ }^{t}, v_{z}{ }^{t}\right)$ the objects in the scene.

The derivatives of the matching function are canceled, in relation to $d_{x}$ and to $d_{y} \rightarrow$ Optical flow computation $=$ (Apparent field of movement) 


$$
\begin{aligned}
\left(v_{x}^{t}, v_{y}^{t}\right) & =\arg \min _{\left(\delta x, \delta_{y}\right) \in K} A(\delta x, \delta y) \\
& =\arg \min _{\left(\delta x, \delta_{y}\right) \in K} \sum_{(x, y) \in B}(I(x, y, t)-I(x+\delta x, y+\delta y, t+1))^{2}
\end{aligned}
$$

Assuming that the displacement $\left(d_{x}, d_{y}\right)$ is small, then the Taylor expansion to order 1 of I becomes:

$$
I(x+\delta x, y+\delta y, t+1) \approx I(x, y, t)+\frac{\partial I}{\partial x} \delta x+\frac{\partial I}{\partial y} \delta y+\frac{\partial I}{\partial t}
$$

Therefore the matching function A becomes:

$$
\begin{aligned}
& A(\delta x, \delta y) \approx \sum_{(x, y) \in B}\left(\frac{\partial I}{\partial x} \delta x+\frac{\partial I}{\partial y} \delta y+\frac{\partial I}{\partial t}\right)^{2} \\
& A(\delta x, \delta y) \approx \sum_{(x, y) \in B}\left(\frac{\partial I}{\partial x} \delta x+\frac{\partial I}{\partial y} \delta y+\frac{\partial I}{\partial t}\right)^{2}
\end{aligned}
$$

The method of Lucas and Kanade keeps luminance between two successive frames with a constant on the optical flow in the vicinity of $\Omega$ in the pixel will calculate the displacement.

The function to be minimized is:

$$
E_{l k}=\sum_{\Omega}\left[\frac{\partial E(x, y, t)}{\partial x} u(t)+\frac{\partial E(x, y, t)}{\partial y} v(t)+\frac{\partial E(x, y, t)}{\partial t}\right]^{2}
$$

Hence the solution of the maximum likelihood estimation allows least squares, then it must be resolved in the vicinity of $\Omega$ this equation:

$$
A=\ldots\left[\begin{array}{l}
E_{x 1} E_{y 1} \\
E_{x 2} E_{y 2} \\
\cdots \ldots \\
E_{x n} E_{y n}
\end{array}\right], b=\left[\begin{array}{l}
E_{t 1} \\
E_{t 2} \\
\cdots \\
E_{t n}
\end{array}\right]
$$

With $x_{1}, x_{2}, \ldots, x_{n}$ are pixels in the windows $\Omega$ and $E_{x 1}, E_{x 2}, \ldots, E_{x n}$ is the partial derivatives of the image I.

$$
\text { Then }\left[\begin{array}{l}
u \\
v
\end{array}\right]=\left(A^{t} A\right)^{-1} A^{T} b
$$

Or $A^{t}$ is equivalent to:

$$
\begin{gathered}
G=\left[\begin{array}{c}
\sum_{\Omega} E^{2} \sum_{\Omega} E_{x} E_{y} \\
\sum_{\Omega} E_{x} E_{y} \sum_{\Omega} E_{y}^{2}
\end{array}\right], \eta=\left[\begin{array}{l}
\sum_{\Omega} E_{t} E_{y} \\
\sum_{\Omega} E_{t} E_{y}
\end{array}\right] \\
\text { Then }\left[\begin{array}{l}
u \\
v
\end{array}\right]=C^{-1 . n}
\end{gathered}
$$

But we experienced a problem opening (zero gradients) as the pixel is in the part where the light intensity is constant when calculating the least-squares estimation gives aberrant.

This problem can be eliminated by using the quadratic regularization technique where the equation becomes:

$$
E \sum_{\Omega}\left[\vee E \cdot \bar{w}+E_{t}\right]^{2}+\alpha|w|^{2}
$$

The final function becomes:

$$
\left[\begin{array}{l}
u \\
v
\end{array}\right]=\left(A^{T} A+\alpha E\right)^{-1} A^{T} b
$$

With $\alpha$ is the regularity of the solution.
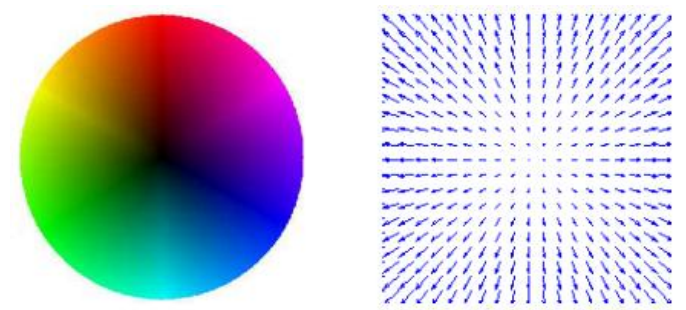

Fig. 3. Velocity field representation.

In the color map of velocity vectors (see Fig. 3) are represented by the colors contained within the circle. Each vector is encoded by the color that indicates placing its origin at the center of the circle. The intensity varies from black to full color until a maximum speed for display (White).

For visual were representing the route of the optical flow field are used of the vectors in the original image superimposed speeds, can also be used to map a color to represent the direction of flow as well as its intensity (see Fig. 4).
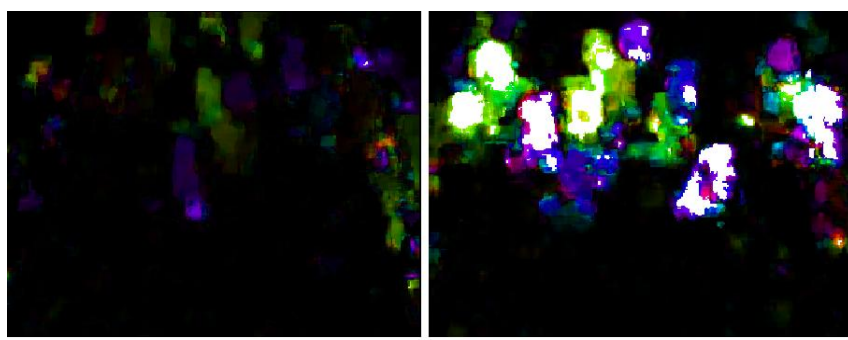

Fig. 4. Real-time estimation of Optical Flow for the detection of abnormal behavior.

\section{EXPERIMENTS AND DISCUSSION}

To evaluate our approach, we tested our algorithm on a video scene all throughout the surveillance. In this scene we mean by abnormal movement all movement suddenly. To detect the abnormal movement were calculated light intensity obtained after the application of the Lucas Kanade algorithm.

If the light intensity (see Fig. 5) is very important that is greater than 2000 then we can deduce that there is an abnormal movement, otherwise if the value of the intensity is less than 1000, we can consider it as a normal movement (experimental results). 


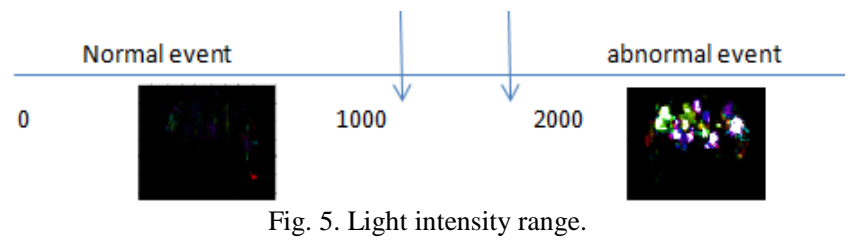

Our approach is tested on the data set publicly available videos from the University of Minnesota [15] normal and abnormal crowd. The UMN Dataset contains a video of 11 scenarios currency on three different indoor and outdoor stages. Each video contains a portion consisting of normal behavior and ends with abnormal behavior (see Fig. 6).
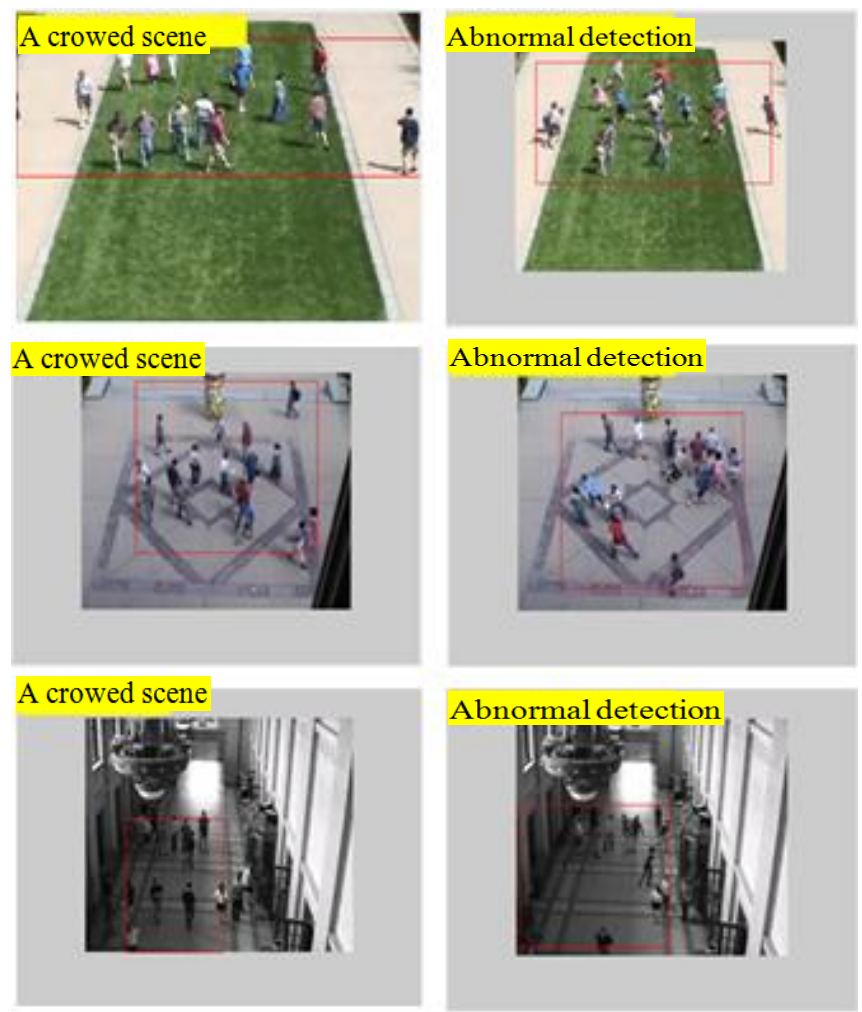

Fig. 6. Abnormal events detection in a crowd.

To evaluate our approach, we calculate the real positve rate (TPR) and the false positive rate (FPR) in the movie, using the ROC curve (see Fig. 7).

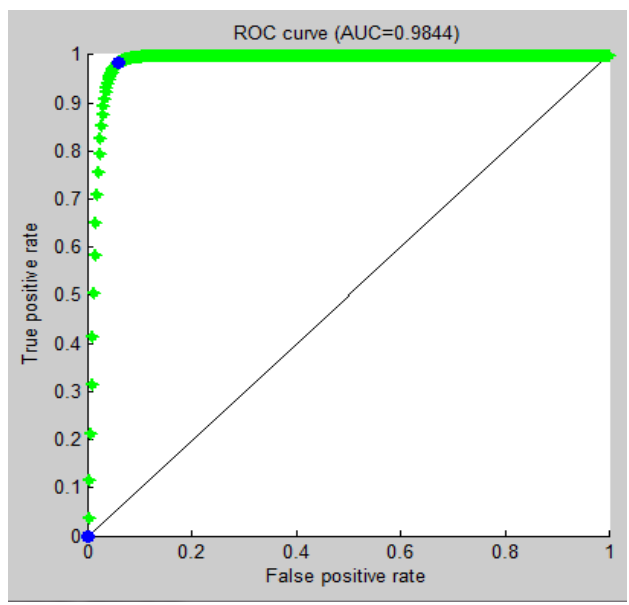

Fig. 7. ROC curves for the detection of abnormal movements.

We compare our approach with social force model, optical flow [6] and streakline potentials [7] (see Table I); these results show that for the detection of abnormal events, our approach is effective relative to other approaches.

TABLE I: COMPARISON OF OUR APPROACH WITH OTHER METHODS FOR THE DETECTION OF ABNORMAL EVENTS THROUGHOUT UMN DATA

\begin{tabular}{ll}
\hline \hline Method & Area under ROC \\
Social Force[6] & 0.96 \\
Optical flow[6] & 0.84 \\
Streakline potentials[7] & 0.9 \\
Ours & 0.984 \\
\hline \hline
\end{tabular}

\section{CONCLUSION}

In this paper we propose a new method for monitoring and for the detection of abnormal events; we have used the criterion of light intensity that corresponds to the rate of movement of individuals to detect abnormal movement. The benefits for our proposed method is that it no longer contains learning phase and despite that our approach provides a method for the temporal tracking of individuals over time we do not need to follow those in phase anomaly Detection.

\section{ACKNOWLEDGMENT}

The authors would like to acknowledge the financial support of this work by grants from General Direction of Scientific Research (DGRST), Tunisia, under the ARUB program.

\section{REFERENCES}

[1] P. P. Chaturvedi, A. S. Rajput, and A. Jain, "Video object tracking based on automatic background segmentation and updating using RBF neural network," International Journal of Advanced Computer Research, vol. 3, p. 866, June 2013.

[2] J. Kim and K. Grauman, "Observe locally, infer globally: A space-time MRF for detecting abnormal activities with incremental updates," in Proc. IEEE Conference on Computer Vision and Pattern Recognition, June 2009, pp. 2921-2928.

[3] B. Zhan, D. N. Monekosso, P. Remagnino, S. A. Velastin, and X. Il-Qun, "Crowd analysis: Asurvey," Machine Vision and Applications, pp. 345-357, October 2008.

[4] I. Rodrigues de Almeida and C. R. Jung, "Change detection in human crowds," in Proc. Conference on Graphics, Patterns and Images, August 2013, pp. 63-69.

[5] V. Mahadevan, W. Li, V. Bhalodia, and V. Masconcelos, "Anomaly detection in crowded scenes," in Proc. IEEE Conference on Computer Vision and Pattern Recognition, June 2010, pp. 1975-1981.

[6] S. Kamijo, Y. Matsushita, K. Ikeuchi, and M. Sakauchi, "Traffic monitoring and accident detection at intersections," in Proc. IEEE Transactions on Intelligent Transportation Systems, June 2000, vol. 2, pp. 108-118.

[7] N. Friedman and S. Russell, "Image segmentation in video sequences: A probabilistic approach," in Proc. Conference on Uncertainty in Artificial Intelligence, 1997, pp. 175-181.

[8] J. Wang and Z. Xu, "Texture-based homogeneity analysis for crowd scene modelling and abnormality detection," in Proc. International Conference on Autonomic Computing, 2014.

[9] M. Tipping and C. Bishop, Mixtures of Probabilistic Principal Component Analyser, MIT Press, pp. 443-482, June 2006.

[10] R. Mehran, B. E. Moore, and M. Shah, "A streakline representation of flow in crowded scenes," ECCV (3), Springer, pp. 439-452, 2010.

[11] A. Adam, E. Rivlin, I. Shimshoni, and D. Reinitz, "Robust real-time unusual event detection using multiple fixed-location monitors," IEEE Transactions on Pattern Analysis and Machine Intelligence, vol. 30, pp. 555-560, March 2008.

[12] A. Finamore, M. Mellia, M. Meo, M. M. Munafo, and D. Rossi, "Experiences of Internet traffic monitoring with tstat," in Proc. Network IEEE, May 2011, vol. 25.

[13] R. Mehran, A. Oyama, and M. Shah, "Abnormal crowd behavior detection using social force model," in Proc. IEEE Conference on Computer Vision and Pattern Recognition, June 2009, pp. 935-942. 
[14] L. Spinello and K. O. Arras, "People detection in RGB-D data," in Proc. IEEE/RSJ International Conference on Intelligent Robots and Systems, September 2011, pp. 3838-3843.

[15] A. Adam, E. Rivlin, I. Shimshoni and D. Reinitz, "Robust real-time unusual event detection using multiple fixed-location monitors," IEEE Transactions on Pattern Analysis and Machine Intelligence, vol. 30, pp. 555-560, March 2008.

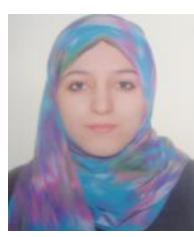

Mariem Gnouma received the license degree in computer science from the Faculty of Sciences of Gabes (FSG), and the master degree of computer science and multimedia from the Higher Institute of Computer and Multimedia of Gabes (ISIMG) respectively in 2012, 2015.

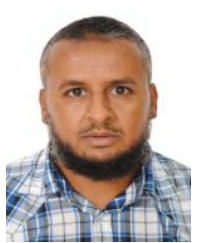

Ridha Ejbali received the Ph.D. degree in computer engineering, the Master degree and computer engineer degree from the National Engineering School of Sfax Tunisia (ENIS) respectively in 2012, 2006 and 2004.

He was an assistant technologist at the Higher Institute of Technological Studies, Kebili Tunisia since 2005. He joined the Faculty of Sciences of Gabes Tunisia (FSG) where he is an assistant in the Department of Computer Sciences since 2012.
His research area is now in pattern recognition and machine learning using Wavelets and Wavelet networks theories.

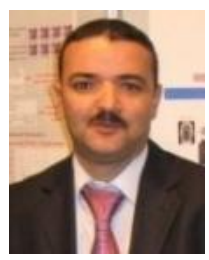

Mourad Zaied received the HDR, the Ph.D. degrees in computer engineering and the master degree of science from the National Engineering School of Sfax respectively in 2013, 2008 and in 2003. He obtained the degree of computer engineer from the National Engineering School of Monastir in 1995. Since 1997 he served in several institutes and faculties in university of Gabes as teaching assistant. He joined in 2007 the National Engineering School of Gabes (ENIG) as where he is currently an associate professor in the Department of Electrical Engineering. His research interests include computer vision and image and video analysis. These research activities are centered around wavelets and wavelet networks and their applications to data classification and approximation, pattern recognition and image, audio and video coding and indexing. He was the chair of the Workshop on Intelligent Machines: Theories \& Applications (WIMTA II 2009) and he organized two winter schools on wavelet and its applications (2005) and on Matlab toolkits (2004). 